\title{
Análise dos efeitos da cinesioterapia e da hidrocinesioterapia em indivíduos com artrite reumatoide: ensaio clínico randomizado
}

\author{
Analysis of effects of kinesiotherapy and hydrokinesiotherapy on individuals with \\ rheumatoid arthritis: randomized clinical trial
}

\begin{abstract}
Análisis de los efectos de la cinesioterapia y de la hidrocinesioterapia en individuos con artritis reumatoide: ensayo clínico aleatorizado
\end{abstract}

Bruna da Silva Pavan1, Gabriela Silva Garcia1, Talita Zonta1, Roberta Pez Fagundes¹, Tamiris Natália Chiossi ${ }^{1}$, Jéssica da Silva Pinheiro ${ }^{1}$, Karine Demartini ${ }^{1}$, Márcia de Oliveira Siqueira ${ }^{1}$, Ângela Vieira da Silva Oliveira ${ }^{1}$, Eduarda Grigoletto Althaus ${ }^{1}$, Afonso Vazzoler ${ }^{1}$, Matheus Santos Gomes Jorge $^{1 *}$, Lia Mara Wibelinger ${ }^{1}$.

\section{RESUMO}

Objetivo: Comparar os efeitos da cinesioterapia e da hidrocinesioterapia sobre a força de preensão palmar, a dor e a qualidade de vida em indivíduos com artrite reumatoide. Métodos: Ensaio clínico randomizado, com 12 indivíduos com artrite reumatoide. O G1 $(n=05)$ realizou 10 sessões de cinesioterapia e o $G 2(n=07)$ realizou 15 sessões de hidrocinesioterapia. Resultados: o G1 apresentou aumento da força de preensão bilateral e melhora dos domínios dor e vitalidade da qualidade de vida $(p<0,05)$. O G2 apresentou aumento da força de preensão na mão esquerda, diminuição da intensidade da dor e melhora do domínio estado geral de saúde da qualidade de vida $(p<0,05)$. Observou-se superioridade do $G 2$ em relação ao $G 1$ no alívio da intensidade da dor $(p<0,05)$. Conclusão: A cinesioterapia melhora a força de preensão palmar e a qualidade de vida, enquanto a hidrocinesioterapia melhora a força de preensão palmar, a qualidade de vida e a intensidade da dor, em indivíduos com artrite reumatoide. A hidrocinesioterapia produz melhores efeitos quando comparada à cinesioterapia no alívio da dor destes indivíduos.

Palavras-Chave: Artrite reumatoide, Fisioterapia, Hidroterapia, Exercício, Reabilitação.

\begin{abstract}
Objective: To compare the effects of kinesiotherapy and hydrokinesiotherapy on palmar grip strength, pain and quality of life in individuals with rheumatoid arthritis. Methods: Randomized clinical trial of 12 individuals with rheumatoid arthritis. $\mathrm{G} 1(\mathrm{n}=05)$ performed 10 sessions of kinesiotherapy and $\mathrm{G} 2(\mathrm{n}=07)$ performed 15 sessions of hydrokinesiotherapy. Results: G1 presented increased bilateral grip strength and improvement of domains pain and vitality of quality of life $(p<0.05)$. G2 presented increase of the grip strength in the left hand, decrease of pain intensity and improvement of domain general health status of the quality of life $(p<0.05)$. G2 was superior to $\mathrm{G} 1$ in relieving pain intensity $(p<0.05)$. Conclusion: Kinesiotherapy improves palmar grip strength and quality of life, while hydrokinesiotherapy improves palmar grip strength, quality of life, and pain intensity in individuals with rheumatoid arthritis. Hydrokinesitherapy produces better effects when compared to kinesiotherapy in relieving the pain of these individuals.
\end{abstract}

Key words: Rheumatoid arthritis, Physical therapy specialty, Hydrotherapy, Exercise, Rehabilitation.

${ }^{1}$ Universidade de Passo Fundo (UPF), Passo Fundo-RS. *E-mail: matheussgjorge@gmail.com

SUBMETIDO EM: 5/2019

ACEITO EM: 6/2019

PUBLICADO EM: 7/2019

REAS/EJCH | Vol. Sup.26 | e880 | DOI: https://doi.org/10.25248/reas.e880.2019 Página 1 de 10 


\section{RESUMEN}

Objetivo: Comparar los efectos de la cinesioterapia y de la hidrocinesioterapia sobre la fuerza de asimiento palmar, el dolor y la calidad de vida en individuos con artritis reumatoide. Métodos: Ensayo clínico aleatorizado, con 12 individuos con artritis reumatoide. El $G 1(n=05)$ realizó 10 sesiones de cinesioterapia y el G2 ( $n=07)$ realizó 15 sesiones de hidrocinesioterapia. Resultados: el G1 presentó aumento de la fuerza de asimiento bilateral y mejora de los dominios dolor y vitalidad de la calidad de vida $(p<0,05)$. El G2 presentó aumento de la fuerza de asimiento en la mano izquierda, disminución de la intensidad del dolor y mejora del dominio estado general de salud de la calidad de vida $(p<0,05)$. Se observó superioridad del $G 2$ en relación al $\mathrm{G} 1$ en el alivio de la intensidad del dolor $(p<0,05)$. Conclusión: La cinesioterapia mejora la fuerza de asimiento palmar y la calidad de vida, mientras que la hidrocinesioterapia mejora la fuerza de asimiento palmar, la calidad de vida y la intensidad del dolor, en individuos con artritis reumatoide. La hidrocinesioterapia produce mejores efectos cuando se compara con la cinesioterapia en el alivio del dolor de estos individuos.

Palabras clave: Artritis Reumatoide, Fisioterapia, Hidroterapia, Ejercicio, Rehabilitación.

\section{INTRODUÇÃO}

A artrite reumatoide (AR) é uma doença reumática autoimune, inflamatória, crônica e sistêmica, cuja principal característica é a inflamação persistente da membrana sinovial articular, resultando em erosão óssea, destruição da cartilagem, edema e deformidades articulares, cursando com dores persistentes. Manifesta-se simetricamente nos tecidos, nos órgãos e, principalmente, nas articulações periféricas (WIBELINGER LM, 2014; WIBELINGER LM, et al., 2015).

Sua ocorrência pode ser observada em todos os grupos étnicos, com prevalência de, aproximadamente, em $1 \%$ da população brasileira, similar à literatura mundial. Associa-se mais frequentemente ao gênero feminino em uma proporção de 2-3:1 em relação ao gênero masculino. A faixa etária acometida pode variar, sendo que o pico ocorre na idade adulta entre os 20-40 e 60 anos. Sua incidência aumenta com a idade (WIBELINGER LM, 2014).

O quadro clínico da AR é caracterizado pelas fortes dores matinais ou noturnas nas articulações interfalangeanas proximais das mãos, metacarpo e metatarsofalangeanas, punhos, ombros e joelhos. Outras manifestações como o edema simétrico de tecidos moles em áreas articulares, nódulos reumatoides, rigidez matinal ou após períodos prolongados de imobilização com pelo menos uma hora de duração, fadiga, malestar, diminuição da força e resistência muscular e descondicionamento físico, também são identificados nestes indivíduos. Os exames laboratoriais evidenciam a presença de fator reumatoide e os exames radiográficos erosões e/ou osteopenia periarticular nas articulações das mãos e punhos (WIBELINGER LM, 2014).

Visto o íntimo e preferencial envolvimento das articulações distais dos membros superiores, os indivíduos com AR podem apresentar redução da sua força de preensão palmar (FPP) por conta do desuso do membro acometido, decorrente da degeneração articular e do desequilíbrio ligamentar e muscular oriundos da inflamação persistente (IOP RR, et al., 2015). Além da influência sobre a FPP, esta população tende a apresentar impacto na sua qualidade de vida (QV) resultante das dores e outros agravos musculoesqueléticos apresentados, se comparado aos indivíduos sem a doença (MYRA RS, et al., 2015a; AZEVEDO AFB, et al.,2015).

Os objetivos de tratamento para AR são aliviar a dor, diminuir o edema e a rigidez articular, fortalecer a musculatura (sobretudo das mãos), aumentar a amplitude de movimento articular e a flexibilidade dos tecidos, melhorar o condicionamento aeróbico, prevenir incapacidades e promover a QV. Dentre os recursos terapêuticos, os estudos têm apresentado a cinesioterapia e a hidrocinesioterapia como alternativas no manejo e combate de tais problemas enfrentados por estes indivíduos (WIBELINGER LM, 2015; MIOTTO C, et al., 2013). 
A cinesioterapia, que é a terapia por meio do exercício físico ativo ou passivo, pode apresentar benefícios interessantes para indivíduos com AR, tais como um efeito cardiovascular protetor, melhorar em aspectos clínicos da doença (incluindo reduções na inflamação), melhora dos aspectos psicológicos aos indivíduos, entre outros, sem exacerbar os sintomas da doença (METSIOS GM, et al., 2013). Já a hidrocinesioterapia, que a reabilitação física em ambiente aquático, é tida como um tratamento seguro e eficaz. Quando comparado ao tratamento convencional em solo, os benefícios da água são otimizam a melhora da amplitude de movimento articular, da força muscular, da capacidade cardiovascular e do equilíbrio postural, com consequente evolução da capacidade funcional do indivíduo com AR (ROSA CGS, et al., 2015).

Portanto, o objetivo deste estudo foi comparar os efeitos da cinesioterapia e da hidrocinesioterapia sobre a FPP, a dor e a QV em indivíduos com AR.

\section{MÉTODOS}

Este foi um estudo do tipo ensaio clínico randomizado, cujo mesmo foi aprovado pelo Comitê de Ética em Pesquisa em Seres Humanos da Universidade de Passo Fundo sob protocolo no 348.381 e está de acordo com resolução 466/2012 do Conselho Nacional de Saúde (CNS). A população foi composta por 17 indivíduos diagnosticados com AR, que estavam na lista de espera para tratamento do Setor de Reumatologia da Clínica de Fisioterapia, localizada no complexo da Faculdade de Educação Física e Fisioterapia, da Universidade de Passo Fundo (Passo Fundo, Rio Grande do Sul, Brasil). Estes foram selecionados por meio de uma amostragem não-probabilística, por conveniência.

Como critérios de inclusão foram selecionados indivíduos com diagnóstico clínico de AR há pelo menos dois anos, que não estivessem realizando tratamento fisioterapêutico ou de qualquer outra natureza reabilitativa (exceto tratamento medicamentoso) há pelo menos três meses, que não estivessem em período agudo da doença e com capacidade física e psíquica para compreender a dinâmica das avaliações e dos exercícios. Foram excluídos os indivíduos com histórico de AR juvenil, que passaram por procedimentos cirúrgicos prévios por contra da doença, que apresentassem outra forma de artrite (osteoartrite, hemofílica, reativa, psoriática) ou que não realizassem o número de sessões proposta pelo estudo.

Perante os critérios de inclusão e exclusão pré-estabelecidos, todos os indivíduos estariam aptos a participar do estudo. Entrou-se em contato via telefonema com os sujeitos para marcar uma entrevista e avaliação inicial com os mesmos.

Os indivíduos foram recebidos por dois avaliadores que apresentaram-Ihes o Termo de Consentimento Livre e Esclarecido, mediante prévia explicação e esclarecimento de dúvidas. Todos os convocados assinaram, concordando em participar do estudo. Após, os indivíduos foram submetidos a uma avaliação inicial das condições demográfica e físicas dos indivíduos, cuja mesma consistiu na coleta de dados (nome, idade, endereço, profissão, entre outros) com um questionário elaborado pelos próprios pesquisadores. Os desfechos analisados foram a FPP, mensurada por meio do teste de dinamometria manual, a dor, por meio da escala visual analógica da dor, e a QV, por meio do questionário Medical Outcomes Study 36 - Item ShortForm Health Survey (SF-36). Com exceção da coleta de dados, as demais avaliações foram realizadas no primeiro dia e no último dia de encontro com os indivíduos.

A força de preensão palmar foi mensurada por meio da dinamometria manual com um dinamômetro manual da marca Kratos ${ }^{\circledR}$. O procedimento de coleta seguiu as diretrizes da Sociedade Americana de Terapeutas da Mão para realização do teste. Foram realizadas três tentativas e, posteriormente, considerada a média aritmética entre as mesmas. O intervalo entre as mensurações foi de um minuto (JORGE, et al., 2019).

A escala visual analógica da dor é um instrumento, como o próprio nome sugere, destinado a avaliar a dor do indivíduo no momento de sua aplicação. Trata-se de uma escala numérica com escores que varia de zero (nenhuma dor) a 10 (pior dor imaginável). Solicita-se ao indivíduo avaliado que indique, quantitativamente, a

REAS/EJCH | Vol. Sup.26 | e880 | DOI: https://doi.org/10.25248/reas.e880.2019 Página $\mathbf{3}$ de $\mathbf{1 0}$ 
intensidade da dor presente naquele momento (MOREIRA I, et al., 2017). Quanto mais elevado o número indicado, maior é a intensidade da dor experimentada pelo indivíduo.

O questionário Medical Outcomes Study 36 - Item Short-Form Health Survey destina-se a avaliar a QV do indivíduo. Trata-se de uma escala formada por 36 itens, reunidos nos componentes físico e mental e que já foi traduzida para o português e adequada às condições socioeconômicas e culturais da população brasileira (CICONELLI RM, et al., 1999). O componente físico é composto pelos domínios capacidade funcional (10 itens), aspectos físicos (4 itens), dor (2 itens) e estado geral de saúde (5 itens). Já o componente mental abrange os domínios vitalidade (4 itens), aspectos sociais (2 itens), aspectos emocionais ( 3 itens) e saúde mental ( 5 itens). O escore final para cada domínio pode variar de uma pontuação mínima de zero ponto (que corresponde ao pior estado geral de saúde relacionado à QV) a uma pontuação máxima de 100 pontos (que corresponde ao melhor estado geral de saúde relacionado à QV) (MOREIRA I, et al., 2017). .

Após a avaliação, os indivíduos foram distribuídos, por sorteio, a compor o grupo 1 (G1), cujo mesmo realizaria um plano de intervenção cinesioterapêutica, e o grupo 2 (G2), que realizaria um plano de intervenção hidrocinesioterapêutica.

Nestas condições, o G1 foi composto por 07 indivíduos que realizaram 10 sessões de cinesioterapia em grupo, com frequência de duas vezes por semana, durante cinco semanas, com duração média de 50 minutos. Já o G2 foi composto por 10 indivíduos que realizaram 15 sessões de hidrocinesioterapia em grupo, com frequência de duas vezes por semana, durante sete semanas e um dia, com duração de aproximadamente 50 minutos cada sessão e em grupo.

Com base em outro estudo (MYRA RS, et al. 2015a), o programa cinesioterapêutico foi delineado e adaptado visando efeitos sobre a FPP, a dor e a QV dos indivíduos do G1 por meio da seguinte ordem:

1. Alongamentos musculares globais, de modo lento e mantido, da coluna vertebral (regiões cervical, torácica e lombar), membros superiores e inferiores e tronco (30 segundos cada grupo muscular).

2. Fortalecimento dos membros superiores e da cintura escapular com faixas elásticas de diferentes intensidades, por meio das diagonais de membro superior do método Facilitação Neuromuscular Proprioceptiva (03 séries de 10 repetições para cada membro).

3. Fortalecimento da cintura escapular e do membro superior com bastão sem carga associado ao padrão ventilatório profundo (03 ciclos de 05 repetições).

4. Fortalecimento dos músculos posteriores de tronco e membros superiores com faixas elásticas de diferentes intensidades (03 séries de 10 repetições).

5. Fortalecimento dos punhos com faixas elásticas de diferentes intensidades nos movimentos de flexão, extensão, desvio ulnar e desvio radial (03 séries de 15 repetições);

6. Fortalecimento das mãos com fortalecedor de punho e dedos com resistência que variou de 1,4kgf a $4,1 \mathrm{kgf}$ (02 séries de 15 repetições) e bolinhas proprioceptivas com resistências leve e moderada (03 séries de 20 repetições).

7. Exercício de motricidade fina, onde os indivíduos deveriam realizar pegadas em movimento de pinça com todos os dedos em massas de modelar terapêutica (05 minutos realizando este movimento).

8. Exercício de transferência de peso para membros superiores no tatame (03 séries de 05 repetições para cada lado).

9. Exercícios de equilíbrio e propriocepção em plataformas desestabilizadoras, tais como balance pad de espuma, balance pad emborrachado e camas elásticas (03 séries de 10 repetições).

10. Relaxamento final em bolas suíças de $65 \mathrm{~cm}$, alongando-se os músculos do tronco, do pescoço e da cadeia escapular (20 segundos cada grupo muscular).

Com base em um estudo (JORGE MSG, et al., 2016), o programa hidrocinesioterapêutico foi delineado e adaptado visando efeitos sobre a FPP, a dor e a QV dos indivíduos do G2. Com água aquecida a $36^{\circ} \mathrm{C}$, a ordem dos exercícios em meio aquático foi a seguinte:

1. Fase de adaptação, composta por caminhadas em torno da piscina em linha reta, lateral ou cruzada, totalizando duas voltas, e exercício respiratório de padrão diafragmático profundo. 
2. Fase de aquecimento, composta por alongamentos musculares globais, de modo lento e mantido, da coluna vertebral (regiões cervical, torácica e lombar), membros superiores e inferiores e tronco (20 segundos cada grupo muscular) e circundução dos ombros e dos punhos (30 segundos).

3. Fase de fortalecimento, onde foi realizado o fortalecimento das mãos com flutuadores e bolas proprioceptivas, dos músculos adutores e abdutores de ombro com flutuadores, dos músculos do tronco e membros superiores com fluturadores e dos músculos plantiflexores e dorsiflexores dos tornozelos (todos realizados em 03 séries de 10 repetições).

4. Fase de mobilidade e flexibilidade, onde era realizada a rotação de tronco com auxílio de flutuadores em torno do corpo (03 séries de 10 repetições).

5. Fase de equilíbrio postural e propriocepção, onde os indivíduos realizaram a elevação da perna estendida apoiada em um flutuador (02 séries de 07 repetições), realizar apoio unipodal durante 10 segundos resistindo à turbulência da água (01 série de 05 repetições) e realizar descarga de peso látero-lateral nos membros inferiores (03 séries de 10 repetições).

6. Fase de relaxamento, onde os indivíduos ficavam dispostos em fila e realizavam massagem coletiva entre si (05 minutos) e, após, eram submetidos aos princípios da técnica de Watsu, com auxílio de flutuadores na região cervical e nos joelhos (05 minutos).

A reavaliação da intensidade da dor deu-se imediatamente após cada sessão interventiva do G1 e do G2, enquanto a reavaliação da FPP e da QV deu-se após a realização das 10 sessões de cinesioterapia do G1 e das 15 sessões de hidrocinesioterapia do G2. Os dados foram registrados em um programa estatístico onde realizou-se o teste de Komarovsky-Smirnoff para verificar a normalidade. A caracterização da amostra foi realizada por meio de uma estatística descritiva e do teste Qui-Quadrado, enquanto para a análise dos desfechos foi utilizado o teste não paramétrico de Wilcoxon e o Mann-Whitney, admitindo significância estatística quando o valor de $p \leq 0,05$.

\section{RESULTADOS}

Figura 1 - Fluxograma da estratégia de seleção da amostra.

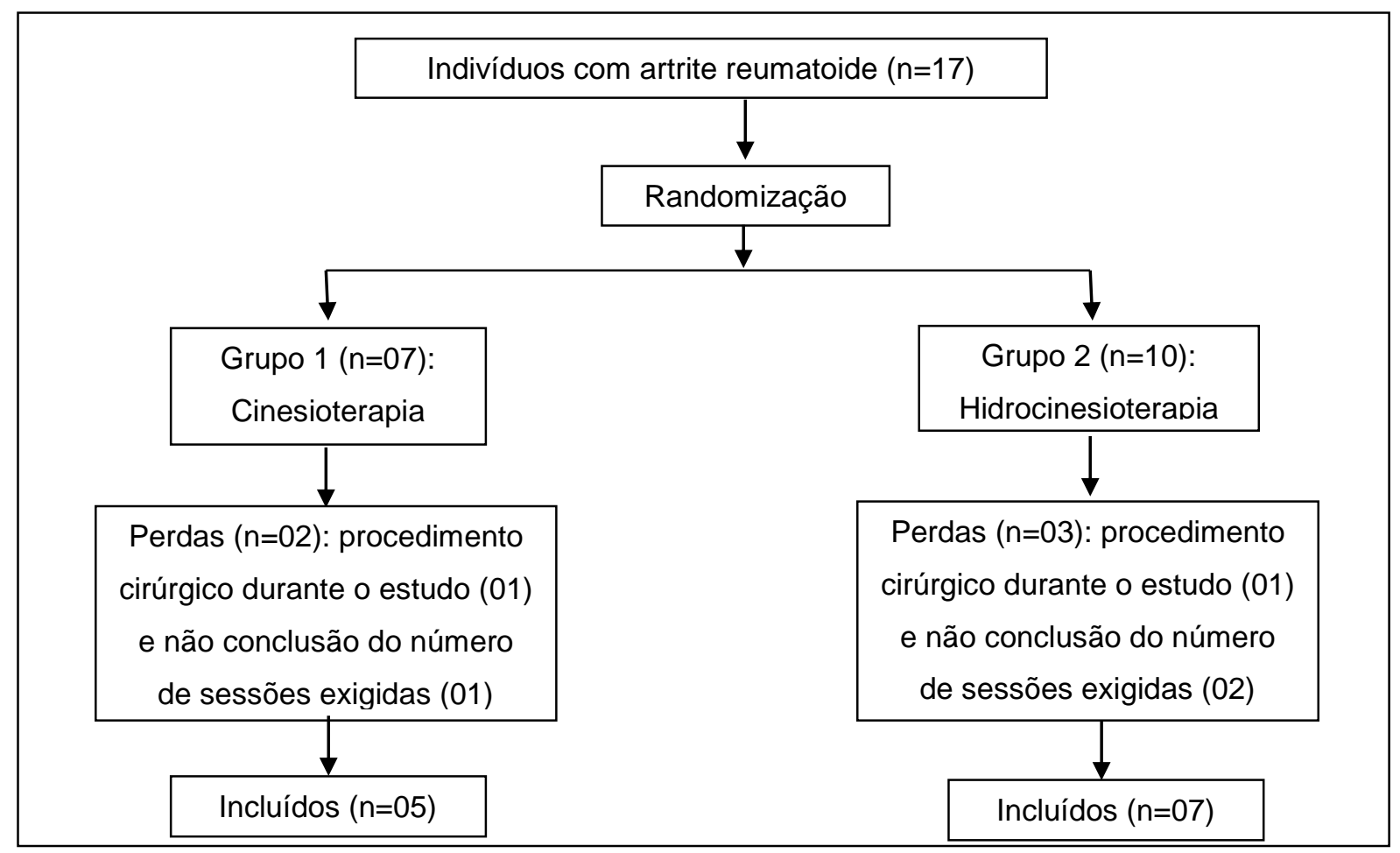

Fonte: Dados da pesquisa, 2018. 
Dos 17 indivíduos inicialmente recrutados cinco foram excluídos (abandono de 29,41\%), sendo dois indivíduos do G1 (um em virtude de um procedimento cirúrgico realizado durante a pesquisa e o outro por não completar o número total de sessões) e três indivíduos do G2 (um em virtude de um procedimento cirúrgico realizado durante a pesquisa e o dois por não completar o número total de sessões). Desta forma, 12 indivíduos com AR compuseram a amostra do presente estudo, com tempo médio de diagnóstico da doença de 15,0 anos e com queixa principal de dor crônica nas mãos (Figura 1).

De acordo com o teste estatístico, a amostra era homogênea, não apresentando diferença na caracterização dos indivíduos entre os grupos (Tabela 1).

Tabela 1 - Caracterização dos pacientes com artrite reumatoide, n=12. Passo Fundo-RS, 2018.

\begin{tabular}{|c|c|c|c|c|c|}
\hline \multirow[b]{2}{*}{ Variável } & \multicolumn{2}{|c|}{ G1 = 05} & \multicolumn{2}{|c|}{ G2 $=07$} & \multirow[b]{2}{*}{$p^{*}$} \\
\hline & $\mathbf{N}$ & $\%$ & $\mathbf{N}$ & $\%$ & \\
\hline Sexo & & & & & 0,200 \\
\hline Masculino & - & - & 01 & $14,3 \%$ & \\
\hline Feminino & 05 & $100 \%$ & 06 & $85,7 \%$ & \\
\hline Idade (média) & \multicolumn{2}{|c|}{54,0 anos } & \multicolumn{2}{|c|}{52,8 anos } & 0,240 \\
\hline Escolaridade & & & & & 0,281 \\
\hline Ensino Fundamental & 03 & $60,0 \%$ & 03 & $43,0 \%$ & \\
\hline Ensino Médio & 01 & $20,0 \%$ & 04 & $57,0 \%$ & \\
\hline Ensino Superior & 01 & $20,0 \%$ & - & - & \\
\hline Estado civil & & & & & 0,558 \\
\hline Com companheiro (a) & 03 & $60,0 \%$ & 02 & $28,6 \%$ & \\
\hline Sem companheiro (a) & 02 & $40,0 \%$ & 05 & $71,4 \%$ & \\
\hline Ocupação laborativa & & & & & 0,558 \\
\hline Ativo no mercado de trabalho & 02 & $40,0 \%$ & 05 & $71,4 \%$ & \\
\hline Inativo no mercado de trabalho & 03 & $60,0 \%$ & 02 & $28,6 \%$ & \\
\hline \multicolumn{6}{|l|}{ Patologias associadas } \\
\hline Sim & 05 & $100 \%$ & 03 & $43,0 \%$ & 0,205 \\
\hline Não & 0 & $0 \%$ & 04 & $57,0 \%$ & \\
\hline Histórico familiar de doença reumática & & & & & 0,293 \\
\hline Sim & 04 & $80,0 \%$ & 03 & 43,0 & \\
\hline Não & 01 & $20,0 \%$ & 04 & $57,0 \%$ & \\
\hline
\end{tabular}

Fonte: Dados da pesquisa, 2018.

Legenda: N (valor absoluto); \% (valor relativo); G1 (Grupo 1); G2 (Grupo 2); *: Teste Qui-quadrado $(p<0,05)$

Observou-se que ambos os programas de intervenção produziram melhora da FPP dos indivíduos, sendo que o G1 apresentou aumento estatisticamente significativo na FPP bilateral, enquanto o G2 apresentou aumento estatisticamente significativo apenas na FPP da mão esquerda (Tabela 2).

Tabela 2 - Força de preensão palmar pré e pós intervenção fisioterapêutica, n=12. Passo Fundo-RS, 2018.

\begin{tabular}{cccc}
\hline & Pré intervenção & Pós intervenção & Valor de $\boldsymbol{p}^{\dagger}$ \\
\hline G1 (n=5) & & & \\
Mão direita & $8,0 \mathrm{~kg}[2,2 ; 12,4]$ & $9,4 \mathrm{~kg}[7,5 ; 8,0]$ & $0,04^{*}$ \\
Mão esquerda & $6,0 \mathrm{~kg}[3,7 ; 12,4]$ & $10,7 \mathrm{~kg}[8,2 ; 17,0]$ & $0,04^{*}$ \\
\hline G2 $(\mathbf{n}=7)$ & & & \\
Mão direita & $6,0 \mathrm{~kg}[4,0 ; 11,0]$ & $8,0 \mathrm{~kg}[5,0 ; 13,0]$ & 0,16 \\
Mão esquerda & $7,0 \mathrm{~kg}[2,0 ; 8,0]$ & $8,0 \mathrm{~kg}[5,0 ; 11,0]$ & $0,01^{*}$ \\
\hline
\end{tabular}

Fonte: Dados da pesquisa, 2018.

Legenda: Mediana [Valor mínimo; Valor máximo]; n (número amostral); * (estatisticamente significativo); G1

(Grupo 1); G2 (Grupo 2); kg (quilograma); † (teste de Wilcoxon). 
Notou-se que ambos as intervenções diminuíram a intensidade do quadro doloroso, porém apenas o G2 apresentou resultados estatisticamente significativos (Tabela 3).

Tabela 3 - Intensidade da dor pré e pós intervenção fisioterapêutica, n=12. Passo Fundo-RS, 2018.

\begin{tabular}{llll}
\hline & Pré intervenção & Pós intervenção & Valor de $\boldsymbol{p}^{\dagger}$ \\
\hline $\mathbf{G 1}(\mathbf{n = 5})$ & $7,0[5,0 ; 7,5]$ & $4,5[4,0 ; 6,0]$ & 0,07 \\
$\mathbf{G} 2(\mathbf{n = 7})$ & $6,0[4,0 ; 8,0]$ & $2,0[1,0 ; 5,0]$ & $0,01^{*}$ \\
\hline
\end{tabular}

Fonte: Dados da pesquisa, 2018.

Legenda: Mediana [Valor mínimo; Valor máximo]; n (número amostral); * (estatisticamente significativo); G1 (Grupo 1); G2 (Grupo 2); † (teste de Wilcoxon).

Ao analisar a QV, observou-se que o G1 apresentou melhora em todos os domínios e o G2 em 05 domínios. Contudo, os resultados estatisticamente significativos foram observados apenas nos domínios dor e vitalidade do G1 e no domínio estado geral de saúde do G2 (Tabela 4).

Tabela 4 - Qualidade de vida pré e pós intervenção fisioterapêutica, $n=12$. Passo Fundo-RS, 2018.

\begin{tabular}{|c|c|c|c|}
\hline & Pré intervenção & Pós intervenção & Valor de $p^{t}$ \\
\hline \multicolumn{4}{|c|}{ Capacidade funcional } \\
\hline $\mathrm{G} 1(\mathrm{n}=5)$ & $25,0[7,5 ; 50,0]$ & $45,0[22,5 ; 55,0]$ & 0,78 \\
\hline G2 $(n=7)$ & $40,0[15,0 ; 80,0]$ & $65,0[25,0 ; 90,0]$ & 0,11 \\
\hline \multicolumn{4}{|c|}{ Limitação por aspectos físicos } \\
\hline $\mathrm{G} 1(\mathrm{n}=5)$ & $0,0[0,0 ; 25,0]$ & $100,0[0,0 ; 100,0]$ & 0,10 \\
\hline $\mathrm{G} 2(\mathrm{n}=7)$ & $0,0[0,0 ; 100,0]$ & $0,0[0,0 ; 100,0]$ & 0,41 \\
\hline \multicolumn{4}{|l|}{ Dor } \\
\hline $\mathrm{G} 1(\mathrm{n}=5)$ & $20,0[10,0 ; 30,5]$ & $41,0[20,0 ; 56,0]$ & $0,03^{*}$ \\
\hline $\mathrm{G} 2(\mathrm{n}=7)$ & $31,0[20,0 ; 42,0]$ & $42,0[22,0 ; 62,0]$ & 0,06 \\
\hline \multicolumn{4}{|c|}{ Estado Geral de Saúde } \\
\hline G1 $(n=5)$ & $40,0[36,0 ; 50,0]$ & $55,0[17,5 ; 62,0]$ & 0,50 \\
\hline $\mathrm{G} 2(\mathrm{n}=7)$ & $32,0[15,0 ; 52,0]$ & $42,0[25,0 ; 67,0]$ & $0,02^{*}$ \\
\hline \multicolumn{4}{|l|}{ Vitalidade } \\
\hline G1 $(n=5)$ & $30,0[27,5 ; 45,0]$ & $65,0[47,5 ; 70,0]$ & $0,04^{*}$ \\
\hline $\mathrm{G} 2(\mathrm{n}=7)$ & $50,0[5,0 ; 75,0]$ & $55,0[30,0 ; 80,0]$ & 0,14 \\
\hline \multicolumn{4}{|c|}{ Aspectos sociais } \\
\hline $\mathrm{G} 1(\mathrm{n}=5)$ & $50,0[37,0 ; 70,1]$ & $65,2[56,3 ; 93,8]$ & 0,08 \\
\hline $\mathrm{G} 2(\mathrm{n}=7)$ & $62,5[37,5 ; 100,0]$ & $62,5[50,0 ; 87,5]$ & 0,33 \\
\hline \multicolumn{4}{|c|}{ Limitação por aspectos emocionais } \\
\hline $\mathrm{G} 1(\mathrm{n}=5)$ & $33,3[0,0 ; 100,0]$ & $66,6[50,0 ; 100,0]$ & 0,50 \\
\hline $\mathrm{G} 2(\mathrm{n}=7)$ & $0,0[0,0 ; 100,0]$ & $100,0[0,0 ; 100,0]$ & 0,15 \\
\hline \multicolumn{4}{|c|}{ Saúde mental } \\
\hline G1 $(n=5)$ & $40,0[28,0 ; 76,0]$ & $68,0[40,0 ; 88,0]$ & 0,13 \\
\hline $\mathrm{G} 2(\mathrm{n}=7)$ & $60,0[16,0 ; 80,0]$ & $56,0[40,0 ; 88,0]$ & 0,20 \\
\hline
\end{tabular}

Fonte: Dados da pesquisa, 2018.

Legenda: Mediana [Valor mínimo; Valor máximo]; n (número amostral); * (estatisticamente significativo); G1 (Grupo 1); G2 (Grupo 2); † (teste de Wilcoxon).

Ao comparar as formas de intervenção, observamos que os indivíduos que realizaram a hidrocinesioterapia apresentaram menores níveis de dor após o tratamento em comparação à cinesioterapia (Tabela 5). 
Tabela 5 - Análise das variáveis intragrupos após a intervenção fisioterapêutica, $n=12$. Passo Fundo-RS, 2018.

\begin{tabular}{llll}
\hline & G1 $(\mathbf{n}=5)$ & G2 $(\mathbf{n}=\mathbf{7})$ & Valor de $^{\boldsymbol{t}}$ \\
\hline FPP mão direita & $9,4 \mathrm{~kg}[0,75 ; 1,80]$ & $8,0 \mathrm{~kg}[0,50 ; 1,30]$ & 0,222 \\
FPP mão esquerda & $10,7 \mathrm{~kg}[8,2 ; 17,0]$ & $8,0 \mathrm{~kg}[5,0 ; 11,0]$ & 0,120 \\
Dor (EVA) & $4,5[4,0 ; 6,0]$ & $2,0[1,0 ; 5,0]$ & $0,038^{*}$ \\
Capacidade funcional & $45,0[22,5 ; 55,0]$ & $65,0[25,0 ; 90,0]$ & 0,087 \\
LAF & $100,0[0,0 ; 100,0]$ & $0,0[0,0 ; 100,0]$ & 0,416 \\
Dor & $41,0[20,0 ; 56,0]$ & $42,0[22,0 ; 62,0]$ & 0,366 \\
Estado geral de saúde & $55,0[17,5 ; 62,0]$ & $42,0[25,0 ; 67,0]$ & 0,935 \\
Vitalidade & $65,0[47,5 ; 70,0]$ & $55,0[30,0 ; 80,0]$ & 0,743 \\
Aspectos emocionais & $65,2[56,3 ; 93,8]$ & $62,5[50,0 ; 87,5]$ & 0,615 \\
LAE & $66,6[50,0 ; 100,0]$ & $100,0[0,0 ; 100,0]$ & 0,862 \\
Saúde mental & $68,0[40,0 ; 88,0]$ & $56,0[40,0 ; 88,0]$ & 0,515 \\
\hline
\end{tabular}

Fonte: Dados da pesquisa, 2018.

Legenda: Mediana [Valor mínimo; Valor máximo]; n (número amostral); * (estatisticamente significativo); G1 (Grupo 1); G2 (Grupo 2(; kg (quilograma); FPP (Força de preensão palmar); EVA (Escala Visual Analógica); LAF (Limitação por aspectos físicos); LAE (limitação por aspectos emocionais); † (teste de Mann-Whitney).

\section{DISCUSSÃO}

Um estudo submeteu um indivíduo com AR à 15 sessões de cinesioterapia baseadas em exercícios globais e específicos para as mãos, e observou-se que, ao final, o sujeito apresentou melhora da FPP das mãos direita e esquerda (aumento de 5,2kgf e 3,2kgf, respectivamente) e diminuição da dor em sete pontos de acordo com a escala visual analógica (MYRA RS, et al., 2015a). Apesar de ter sido adotado um menor tempo de intervenção no presente estudo, o grupo que realizou cinesioterapia também apresentou aumento bilateral da FPP, além da melhora dos domínios dor e vitalidade relacionados a QV. Contudo, não se observou alívio da intensidade da dor, analisada por meio da escala visual analógica.

Em um estudo 20 mulheres com AR e deformidades nas mãos oriundas da doença foram divididas aleatoriamente em $\mathrm{G} 1(n=13)$ que realizaram um programa de exercícios destinados a melhorar a FPP e a coordenação motora da mão, e $G 2(n=7)$ que serviu como controle. $O G 1$ realizou 20 sessões, duas vezes por semana, e exercícios em casa. Após o tratamento, o grupo que realizou exercícios apresentou aumento da FPP e da funcionalidade das mãos, enquanto o controle não apresentou diferença entre as variáveis analisadas (CIMA SR, et al., 2013). Tais constatações corroboram com os achados do presente estudo, onde observou-se que o G1 submetido a cinesioterapia apresentou melhora da FPP.

Neste estudo observou-se que a cinesioterapia produziu benefícios para melhora dos domínios dor e vitalidade na QV. Benefícios semelhantes também foram observados em outro estudo de caso realizado com um indivíduo com $A R$, submetido a 15 sessões de cinesioterapia, onde os resultados evidenciaram uma melhora de cinco domínios da QV, incluindo o domínio dor (MYRA RS, et al., 2015b).

Os efeitos da cinesioterapia mostram-se evidenciados, ainda, em outro estudo onde cinco mulheres com AR foram submetidas ao tratamento fisioterapêutico, baseado nesta abordagem. Os indivíduos realizaram alongamentos, fortalecimentos, exercícios respiratórios, exercícios de motricidade fina, de transferência de peso, de equilíbrio e de propriocepção. Após, constatou-se que, em geral, os indivíduos apresentaram diminuição da intensidade da dor e melhora da QV (SCHNORNBERGER CM et al., 2017). O que assemelhase ao presente estudo, em relação aos resultados apresentados pelo G1.

Estudo de caso de um indivíduo com AR e outras doenças reumáticas, demonstrou que após 30 sessões de fisioterapia, baseada em cinesioterapia e hidrocinesioterapia, apresentou diminuição da dor, aumento da FPP das mãos direita (2,2 kgf) e esquerda (1,2 kgf) e melhora em quatro domínios relacionados à $Q V$, incluindo o estado geral de saúde (JORGE MSG, et al., 2017). 
Apesar de neste estudo, ter sido utilizado os protocolos de cinesioterapia e hidrocinesioterapia separadamente, observou-se alívio da dor, melhora da FPP da mão esquerda e do estado geral de saúde relacionado à $\mathrm{QV}$ dos indivíduos do $\mathrm{G} 2$.

Os resultados encontrados neste estudo com relação à diminuição da intensidade da dor e melhora do domínio estado geral de saúde da QV no G2, corroboram com outro estudo, onde 08 mulheres com AR submetidas a 10 sessões de um programa hidrocinesioterapêutico apresentaram alívio do quadro doloroso com consequente aumento dos escores dos domínios da QV, incluindo o estado geral de saúde (FERREIRA LRF, et al., 2008).

Os efeitos positivos encontrados neste estudo, relacionados à FPP e à dor, também foram encontrados em outro estudo (JORGE MSG, et al., 2016). Os autores utilizaram um protocolo fisioterapêutico combinando um programa de cinesioterapia e um programa de hidrocinesioterapia em um indivíduo com $A R$ e outras doenças reumáticas. Observou-se que a terapia combinada foi eficaz na diminuição da dor de acordo com a escala visual analógica e aumento da FPP bilateral do indivíduo em 1,06kgf no membro superior direito e em $1,56 \mathrm{kgf}$ no membro superior esquerdo. Neste estudo, o G1 (cinesioterapia) foi o único que apresentou aumento na FPP bilateral, enquanto o G2 (hidrocinesioterapia) foi o único que apresentou diminuição da dor de acordo com a escala visual analógica.

Ensaio clínico randomizado realizado com 44 indivíduos com AR divididos em grupo exercício ( $n=21)$, que realizou terapia em meio aquático, e grupo controle $(n=23)$ demonstrou que após 12 semanas de intervenção o tratamento por meio da hidrocinesioterapia (focados na mobilidade articular, resistência/força muscular, equilíbrio/agilidade e flexibilidade) foi efetivo para a melhora em quatro domínios da QV, o alívio da intensidade da dor e o aumento da FPP bilateral (PEDRO JNS, 2017).

Isso vai ao encontro dos resultados obtidos no presente estudo, onde os indivíduos do G2 apresentaram aumento da força da mão esquerda, diminuição do quadro doloroso e melhora no domínio estado geral de saúde relacionado à $\mathrm{QV}$.

Observou-se neste estudo que ambos os grupos apresentaram diminuição da dor de acordo com a escala visual analógica, porém apenas o G2 evidenciou significância estatística. O que vai ao encontro de um estudo (EVERSDEN L, et al., 2007) que submeteu indivíduos com AR a um programa de exercícios físico em solo e em meio aquático, com protocolos semelhantes: 30 minutos de exercícios de aquecimento, mobilidade articular, força muscular e atividades funcionais, durante seis semanas. O grupo que realizou a hidrocinesioterapia apresentou melhores resultados do quadro doloroso imediatamente após as sessões.

Apesar de na literatura já ter sido apresentados estudos que comparem a os efeitos da cinesioterapia e da hidrocinesioterapia separadamente sobre a dor e a QV em indivíduos com AR, como relatado anteriormente, para conhecimento dos autores este pode ter sido o primeiro estudo que demostra tais evidências sobre a FPP deste seleto grupo de indivíduos até o presente momento. Desta forma, sugerem-se a realização de novos estudos com base nos achados apresentados, buscando uma amostra mais expressiva.

O presente estudo apresenta limitações. O número de sessões interventivas não foi homogêneo entre o G1 e ao G2, o que pode ser um fator que tenha contribuído para os efeitos do alívio da dor serem melhores observados no $\mathrm{G} 2$ em relação ao $\mathrm{G} 1$.

Entretanto, os estudos utilizados como referência para delimitar o número de sessões para cada grupo demonstrou que 10 sessões de cinesioterapia (SCHNORNBERGER CM, et al., 2017; SCHNORNBERGER CM, et al., 2018) e 15 sessões de hidrocinesioterapia (JORGE MSG, et al., 2016; JORGE MSG, et al., 2017) são suficientes para produzirem os efeitos sobre as variáveis analisadas neste estudo. Além disso, o tipo de seleção da amostra também pode ter sido um fator limitante deste estudo, o que dificultou na obtenção de uma amostra mais significativa.

Acredita-se que a instituição de um terceiro grupo como controle, ou que utilizasse a combinação da terapia em solo e aquática, poderia ser útil para auxiliar na comparação dos resultados. Tais apontamentos limitam, porém não inviabiliza a geração dos dados finais. 


\section{CONCLUSÃO}

A cinesioterapia produz melhora da FPP e da QV, enquanto a hidrocinesioterapia produz melhora da FPP, da QV e alívio da intensidade da dor, em indivíduos com AR. Ainda, a hidrocinesioterapia produz melhores efeitos quando comparada à cinesioterapia em relação ao alívio da dor, mensurada pela escala visual analógica.

\section{REFERÊNCIAS}

1. AZEVEDO AFB, et al. Quality of life of patients with rheumatoid arthritis under biological therapy. Revista da Associação Médica Brasileira, 2015; 61(2): 126-131.

2. CICONELLI RM, et al. Tradução para a língua portuguesa e validação do questionário genérico de avaliação de qualidade de vida SF-36 (Brasil SF-36). Revista Brasileira de Reumatologia, 1999; 39(3): 143-150.

3. CIMA SR, et al. Strengthening exercises to improve hand strength and functionality in rheumatoid arthritis with hand deformities: a randomized, controlled trial. Rheumatology International, 2013; 33(3): 725-732.

4. EVERSDEN L, et al. A pragmatic randomized controlled trial of hydrotherapy and land exercises on overall well being and quality of life in rheumatoid arthritis. BMC Musculoskeletal Disorders, 2007; 8(1): 23.

5. FERREIRA LRF, et al. Efeitos da reabilitação aquática na sintomatologia e qualidade de vida de portadoras de artrite reumatoide. Fisioterapia e Pesquisa, 2008; 15(2): 136-141

6. IOP RR, et al. Capacidade de produção de força de preensão isométrica máxima em mulheres com artrite reumatoide: um estudo piloto. Fisioterapia e Pesquisa, 2015; 22(1): 11-16.

7. JORGE MSG, et al. Intervenção fisioterapêutica em um indivíduo com artrite reumatoide, lúpus eritematoso sistêmico e síndrome de Sjögren: um relato de caso. Revista de Ciências Médicas e Biológicas, 2016; 15(2): 231 -237.

8. JORGE MSG, et al. Valores da força de preensão palmar em uma população de diferentes faixas etárias. Lecturas: Educación Física y deportes, 2019; 23(249): 56-69.

9. JORGE MSG, et al. Atuação fisioterapêutica em um indivíduo com lúpus associado à artrite reumatoide e à fibromialgia. ABCS Health Sciences, 2017; 42(1): 60-64.

10. METSIOS GS, et al. The role of exercise in the management of rheumatoid arthritis. Expert Review of Clinical Immunology, 2015; 11(10): 1121-1130.

11. MIOTTO C, et al. Tratamento fisioterapêutico das artralgias. Revista Dor, 2013; 14(3): 216-218.

12. MOREIRA I, et al. Métodos de avaliação da coluna vertebral. In: WIBELINGER LM, editora. Disfunções MúsculoEsqueléticas: Prevenção e Reabilitação. 6ª ed. Passo Fundo: Saluz; 2017. p.109-126.

13. MYRA RS, et al. Força de preensão palmar em um indivíduo portador de lúpus eritematoso sistêmico e artrite reumatoide: um estudo de caso. Lecturas: Educación Física y deportes, 2015a; 20(209): 1.

14. MYRA RS, et al. Intervenção cinesioterapêutica na qualidade de vida, dor e força muscular de paciente portador de artrite reumatoide e lúpus eritematoso sistêmico. Relato de caso. Revista Dor, 2015b; 16(2): 153-155.

15. PEDRO JNS. Efeitos da terapia aquática em pessoas com artrite reumatoide. Dissertação (Mestrado em Exercício e Saúde) - Escola de Ciência da Saúde. Universidade de Évora, Évora, 2017; 98 p.

16. ROSA CGS, et al. A eficácia da hidroterapia em pacientes com artrite reumatóide: estudo de revisão. Revista Amazônia Science \& Health, 2015; 3(1): 37-41.

17. SCHNORNBERGER CM, et al. Efeitos da cinesioterapia na força de preensão palmar, na dor e na qualidade de vida de mulheres com artrite reumatoide. Revista de Terapia Ocupacional da Universidade de São Paulo, 2018; 28(3): 325-332.

18. SCHNORNBERGER CM, et al. Intervenção fisioterapêutica na dor e na qualidade de vida em mulheres com artrite reumatoide. Relato de casos. Revista Dor, 2017; 18(4): 365-369.

19. SUZUKI A, YAMAMOTO K. From genetics to functional insights into rheumatoid arthritis. Clinical and Experimental Rheumatology, 2015; 33(4):40-43.

20. WIBELINGER LM, et al. Perfil de uma população portadora de artrite reumatoide em um município do interior do Rio Grande do Sul. Lecturas: Educación Física y deportes, 2015;19(202): 1.

21. WIBELINGER LM. Fisioterapia em Reumatologia. 2ª ed. Rio de Janeiro: Revinter, 2014; 359p. 